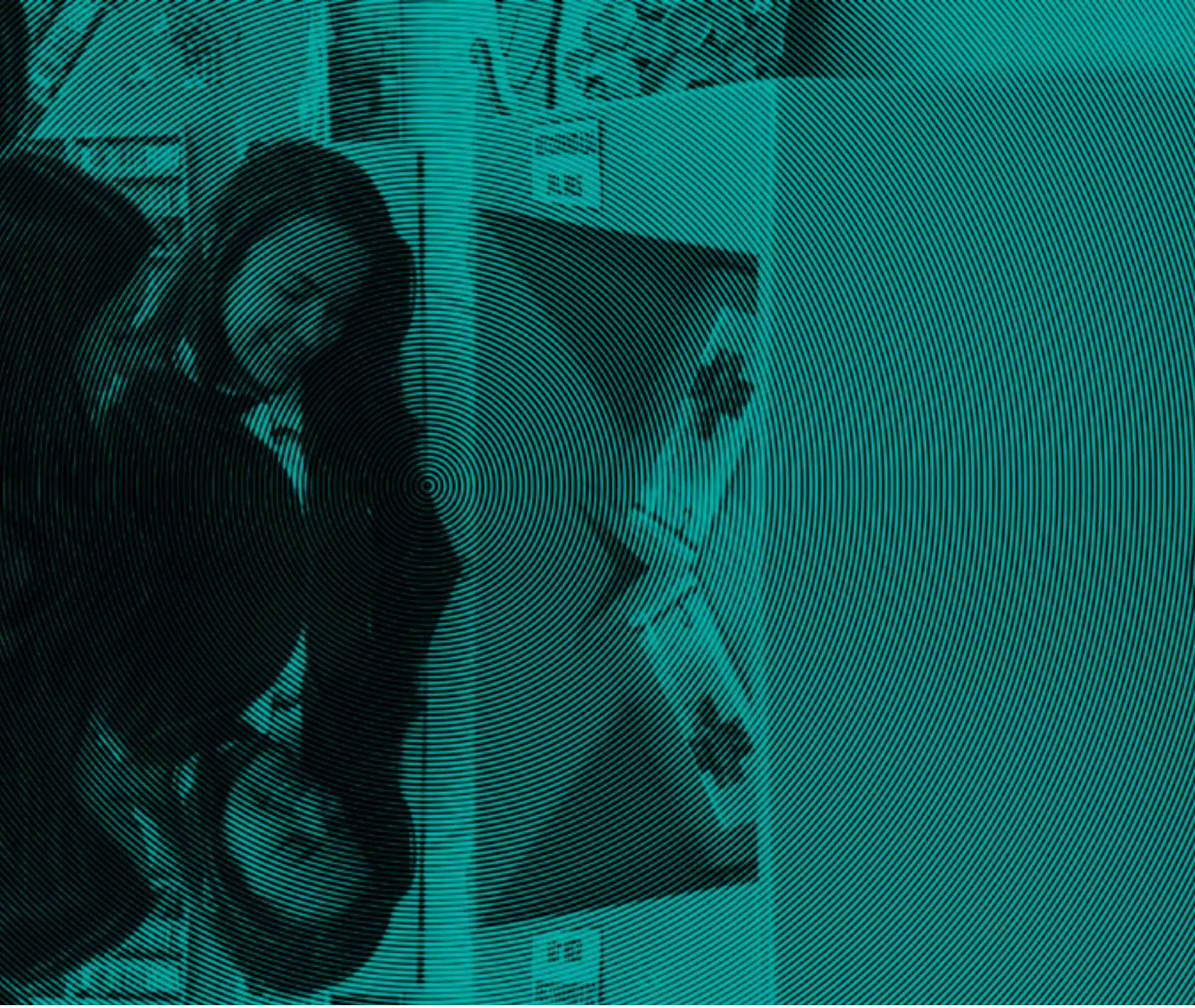

\title{
Hacia una convivencia pacífica en la escuela: percepciones sobre violencia escolar y tramitación de conflictos
}

Towards a Peaceful Coexistence in school: Perceptions of School Violence and Conflict Handling

Para a uma convivência pacífica na escola: Percepções da violência na escola e tramitação dos conflitos

Sindy Paola Díaz Better 
Sindy Paola Díaz Better 1
1. Colombiana. Magíster en Tecnologías de la Información aplicadas a la educación, Universidad Pedagógica Nacional (UPN). Cursando estudios en el Doctorado Interinstitucional en Educación (DIE) - Sede UPN, Bogotá. Docente de la Secretaría de Educación Distrital; correo electrónico: sp.better@gmail.com

Artículo de reflexión basado en investigación.

Fecha de recepciín: Febrero 26 de 2016 / Fecha de aceptación: 16 de septiembre de 2016

\section{Resumen}

Las reflexiones de este artículo provienen de un análisis de percepciones realizado por un grupo de docentes y estudiantes sobre las manifestaciones de violencia escolar y tramitación de conflictos en su institución educativa, problemática que sustenta la tesis presentada por la autora en el Doctorado Interinstitucional en Educación de la Universidad Pedagógica Nacional, Colombia; cuyo punto de partida fue la premisa de que para construir una convivencia pacífica en las escuelas se requiere fomentar relaciones de alteridad y respeto, legitimando las diversas miradas de sus actores sobre las situaciones que los afectan.

\section{Palabras clave: Alteridad,} convivencia pacífica, percepciones de docentes y estudiantes, tramitación de conflictos, violencia escolar.

\section{Summary}

The reflections presented in this article come from analysis of perceptions of a group of teachers and students about manifestations of school violence and conflict handling in their school, problems that supports the thesis of the author, directed by Dr. Luis Fernando Gomez in the Doctorate in Education (Universidad Pedagógica Nacional, Colombia); based on the premise that to build a peaceful coexistence in schools, it is required foster relationships of alterity and respect, legitimizing the diverse views of the school actors about situations that affect them.

\section{Keywords: Alterity, peaceful} coexistence, perceptions of teachers and students, handling conflicts, school violence.

\section{Resumo}

As reflexões apresentadas neste artigo vêm da análise das percepções de um grupo de professores e estudantes sobre as manifestações da violência escolar e a transação dos conflitos na instituição educativa, questões que apóiam a Tese da autora, dirigida pelo Dr. Luis Fernando Gomez no Doutorado Interinstitucional em Educação (Universidade Pedagógica Nacional, Colômbia); com base na premissa que para construir uma convivência pacífica nas escolas, é necessário fomentar relações de alteridade e respeito, legitimando os diferentes olhares de seus atores sobre as situações que os afetam.

Palavras chave: Alteridade, convivência pacífica, percepções dos profesores e estudantes, tramitação dos conflitos, violência escolar. 


\section{Introducción}

$\mathrm{Al}$ hablar de violencia en las escuelas ${ }^{2}$ encontramos una situación generalizada que requiere de miradas contextualizadas para comprenderla y emprender acciones que puedan mitigarla o evitarla. Aunque exista una presencia permanente de los padres de familia y roles de dirección; son los docentes y los estudiantes quienes continuamente se encuentran de frente con manifestaciones que atentan contra la convivencia escolar, por lo que sus percepciones sobre las situaciones de conflicto son fundamentales.

Los programas de intervención y estrategias de manejo de conflictos están dirigidos principalmente a estudiantes, pero, ¿se toman en cuenta sus percepciones?, ¿son comunes las percepciones de docentes y estudiantes frente a las situaciones de violencia escolar que se presentan?, ¿qué estrategias identifican unos y otros?, ¿qué se puede inferir a partir de estas estrategias?, estos y otros interrogantes motivan las reflexiones de este texto. Siguiendo a Mockus y Corzo (2003):

La convivencia se puede caracterizar [...] por una combinación de una alta capacidad reguladora de ley, moral y cultura sobre los individuos, con la capacidad de los propios individuos de celebrar y cumplir acuerdos. Esta combinación produce confianza y se fortalece con ella (p. 14).

Llevando este panorama a la escuela, la convivencia es lo que permite que muchos individuos aprendan en la cotidianidad; sin embargo, los entornos escolares se perciben a veces como espacios homogéneos antes que diversos, al no tener en cuenta que, como sujeto, cada estudiante posee cualidades, capacidades e intereses que lo hacen único; lo que en ciertos casos puede favorecer la aparición de situaciones de conflicto.

Aunque es uno de los pilares de la formación en Bogotá (Colombia), en los últimos años la convivencia pacífica de algunos de sus colegios se ha visto afectada por episodios de violencia que perturban el normal transcurrir de las actividades académicas, hechos expuestos en el informe Clima escolar y victimización en Bogotá 2013 (Ávila, Bromberg, Pérez y Villamil, 2014), un estudio sobre condiciones de seguridad y convivencia para los niños, niñas y jóvenes en los colegios y su entorno; donde se discuten además los

2 En este documento se emplean los términos institución educativa, colegio o escuela indistintamente, para referirse a entidades de carácter público (estatal) o privado, cuya finalidad sea la educación (primaria, secundaria y/o media) de niños, niñas o adolescentes. resultados y aprendizajes de dos encuestas anteriores, realizadas en 2006 y $2011^{3}$.

Entre sus hallazgos, Ávila, et al. (2013) mencionan el empleo de insultos, maltrato verbal y un trato impersonal y de carácter soez entre estudiantes (especialmente en los grados inferiores, pp. 215219), y el maltrato físico intencional (reflejado en golpes, cachetadas, empujones o pellizcos), en porcentajes similares al uso de insultos que causan daño, independientemente de si el colegio es público o privado (pp. 227-232). Estos indicadores también se han hecho presentes en una institución educativa distrital de la Localidad Rafael Uribe Uribe, a través de eventos relacionados con conflictos interpersonales que escalaron en violencia, involucrando incluso el uso de armas blancas como medio de intimidación y agresión.

Frente a esta situación, y con el objetivo de formular una Tesis Doctoral $^{4}$, se realizó un estudio diagnóstico entre los meses de noviembre de 2013 y agosto de 2014, atendiendo al interrogante ¿Qué manifestaciones y situaciones atentan contra la convivencia escolar en esta institución?, para lo cual se acudió a distintas fuentes: Actas de Comisión y Promoción de estudiantes (año 2013); libros observadores de estudiantes (de tres niveles); entrevista a docentes y encuesta a estudiantes de grado sexto.

Las reflexiones que este artículo presenta se desarrollaron a partir del análisis comparativo de las respuestas de los docentes y estudiantes participantes, con el objetivo de dilucidar similitudes y diferencias en sus percepciones de la violencia escolar, las formas de tramitación de conflictos y las estrategias que proponen frente a este fenómeno; lo cual es necesario y pertinente para establecer un panorama general desde sus miradas, y así proponer una reflexión pedagógica sobre la importancia de valorar los puntos de vista de los otros en la construcción de una convivencia pacífica.

3 Entre los resultados se destaca la existencia de hurtos sin violencia, la presencia de manifestaciones de maltrato emocional, acoso escolar, acoso sexual verbal, porte de armas dentro del colegio y consumo de alcohol y drogas ilícitas (Ávila, et al, 2013, pp. 25, 30, 38).

4 Investigación que pretende aportar al campo de estudio de la convivencia escolar desde una perspectiva propositiva; analizando los imaginarios sobre convivencia en el contexto escolar para desarrollar una intervención pedagógica que emplea el lenguaje literario como mediador en la convivencia pacífica. Es dirigida por el Dr. Luis Fernando Gómez Rodríguez (lfgomez@pedagogica. edu.co). 


\section{Apreciaciones sobre la violencia escolar}

En palabras de Camargo (1996), en la escuela se generan espacios de intolerancia, discriminación, exclusión y marginación, dando lugar a brotes de violencia o a espacios propicios para que se manifieste. Frente a esta situación, se deben implementar estrategias que deben ser desarrolladas a partir del conocimiento de la realidad del contexto de una institución educativa. Por su parte, Castro (2010) indica que la reflexión sobre la violencia escolar implica algunas consideraciones como las posibles raíces violentas de la comunidad próxima a la escuela; también señala que no se deben minimizar los episodios de violencia ni juzgarlos como sucesos aislados o accidentales.

Así, propiciar un reconocimiento de las percepciones y prácticas relacionadas con violencia escolar daría indicios sobre los caminos posibles de acción. Así, Henry (2000), luego de analizar algunos de estos aspectos, propone una definición integrada en la que declara que:

La violencia escolar es el ejercicio de poder sobre otros en los entornos escolares, por alguna persona, organismo o proceso social ${ }^{5}$, que niega a aquellos sujetos para que su humanidad pueda hacer diferencia, ya sea reduciéndolos por lo que son, o mediante la limitación de que se conviertan en lo que podrían ser (p. 21).

Esta definición amplía el espectro de la violencia escolar al involucrar otros actores e identificar entornos que también son permeados por las acciones que se llevan a cabo en la escuela. Por ejemplo, gracias a las tecnologías de la información y la comunicación, estamos interconectados a través de múltiples medios, por lo que manifestaciones de índole simbólico como la humillación, también son difundidas a través de ellos.

Para García, Guerrero y Ortiz (2012), la violencia escolar es una de las manifestaciones de otros tipos de violencia del entorno que incluyen conductas como abuso, trasgresión, intimidación, represión, maltrato o humillación, y que muchas veces pasan desapercibidos en diferentes contextos. Estudios internacionales como los de Martínez y Pérez (2005), Arellano (2007), y Powell y Ladd (2010), coinciden en que a veces los brotes de violencia en los colegios son reflejo de procesos y conductas violentas que viven los estudiantes en su entorno.

5 Henry sostiene que al hablar de violencia escolar por lo general se centra la atención en actores como estudiantes y docentes, sin embargo, se han evidenciado relaciones violentas de directivos hacia docentes y estudiantes.
Por otra parte, como mencionan González, Villalobos y Lauretti (2009), se ha hecho énfasis en la necesidad de atender tempranamente expresiones y conductas agresivas, para evitar perturbar a gran escala la convivencia pacífica, esto porque pueden afectar el clima social, que es uno de los factores que puede incidir en los resultados positivos en la educación de los estudiantes (Allodi, 2010) ${ }^{6}$. Teniendo en cuenta estos y otros aportes teóricos, este trabajo asume la violencia escolar como todas las relaciones de violencia cuyo propósito es maltratar, desde el lenguaje o la acción, a cualquier miembro de una comunidad educativa, y que son evidenciadas por prácticas y expresiones en escenarios presenciales o virtuales.

\section{El conflicto escolar y su tramitación}

El conflicto es una situación donde las partes involucradas perciben la existencia de necesidades, intereses, propósitos u objetivos diferentes y a veces incompatibles (Arellano, 2007); esto lleva a que sus pretensiones, deseos e intereses no puedan lograrse simultáneamente, generándose manifestaciones de diversa índole e intensidad. El contexto escolar es un escenario propicio para que se manifiesten conflictos, ya que se establecen distintos tipos de relaciones sociales mediadas por afectos y sentimientos. Lo que debe verse entonces no es la invisibilidad de esas situaciones, sino la manera como se atienden; es decir, cómo lograr que los alumnos puedan expresar sus necesidades personales sin caer en actitudes que les lastimen a sí mismos o a los otros. Tal como señalan Johnson y Johnson (1999): "lo que determina que los conflictos sean constructivos o destructivos no es su existencia, sino el modo en que se los maneja" (p. 29).

En este sentido, interesa el término tramitación, dado que muchas situaciones de conflicto no llegan a resolverse totalmente, por lo que es primordial comprenderlas y tratar de que cambien a estados donde los efectos negativos para los implicados sean mínimos; haciendo la salvedad de que al no lograrse pueden escalar y manifestarse violentamente. El sentido del conflicto como algo que no debe existir en el medio escolar ha sido superado por la

6 En este estudio se analiza la relación entre el clima social que se vive en ambientes educativos, y los resultados positivos en la educación de los estudiantes; manifestando que el sentirse seguro en las escuelas puede disminuir los casos de violencia que se presentan al interior de las mismas. 
idea de un componente inherente a la convivencia, por lo que es necesario que los actores involucrados en el proceso educativo lo utilicen de una forma constructiva.

Los conflictos son muy importantes para el desarrollo psicosocial, por lo que pueden dejar efectos positivos y negativos a largo plazo (Dincyurek y Civelek, 2008). No se trata de evitarlos, sino de favorecer, desde los contextos escolares, el conocimiento y uso de herramientas para un manejo adecuado de los mismos, que permita a quienes intervienen en ellos, tramitarlos de forma no violenta. En Colombia, frente a la necesidad de legislar respecto de la violencia escolar, se promulgó la Ley 1620 del 15 de marzo de $2013^{7}$, que determina lineamientos generales para tratar casos que afectan la convivencia al interior de la instituciones educativas, estableciendo, entre otros medios, La Ruta de Atención Integral para la Convivencia escolar, en la que aparece la mediación y la negociación para aquellas situaciones conflictivas donde no haya daño a la salud física o mental de los involucrados.

$\mathrm{Al}$ ser las instituciones educativas escenarios formativos, se requiere entender el conflicto como una oportunidad de construcción de relaciones que promueven la democracia, por lo que

7 Por medio de la cual se crea el Sistema Nacional de Convivencia Escolar y formación para el ejercicio de los derechos humanos, la educación para la sexualidad y la prevención y mitigación de la violencia escolar. el desarrollo de cualquier plan de acción en favor de la convivencia pacífica, debe demostrar coherencia entre los supuestos teóricos que lo sustenten y las actividades que se propongan, enmarcados siempre en las características particulares inherentes a cada comunidad educativa, por lo que es pertinente reconocer cómo se percibe la tramitación de los conflictos al interior de la misma.

\section{Metodología}

Este trabajo indagó acerca de las percepciones sobre violencia escolar de estudiantes y docentes de un colegio de Bogotá (Colombia), sus manifestaciones más comunes en la institución, la forma de tratar conflictos y las estrategias que proponen para el manejo de este fenómeno; hace parte del diagnóstico realizado con recursos propios entre noviembre de 2013 y agosto de 2014, con el objetivo de aportar a la formulación de una Tesis Doctoral que, en una de sus fases, contempla una intervención pedagógica para la mejora de la convivencia escolar. Se buscó caracterizar y analizar el panorama de violencia escolar desde la mirada de docentes y estudiantes de una institución educativa urbana de carácter público, nivel de secundaria, donde la investigadora viene trabajando desde 2009.

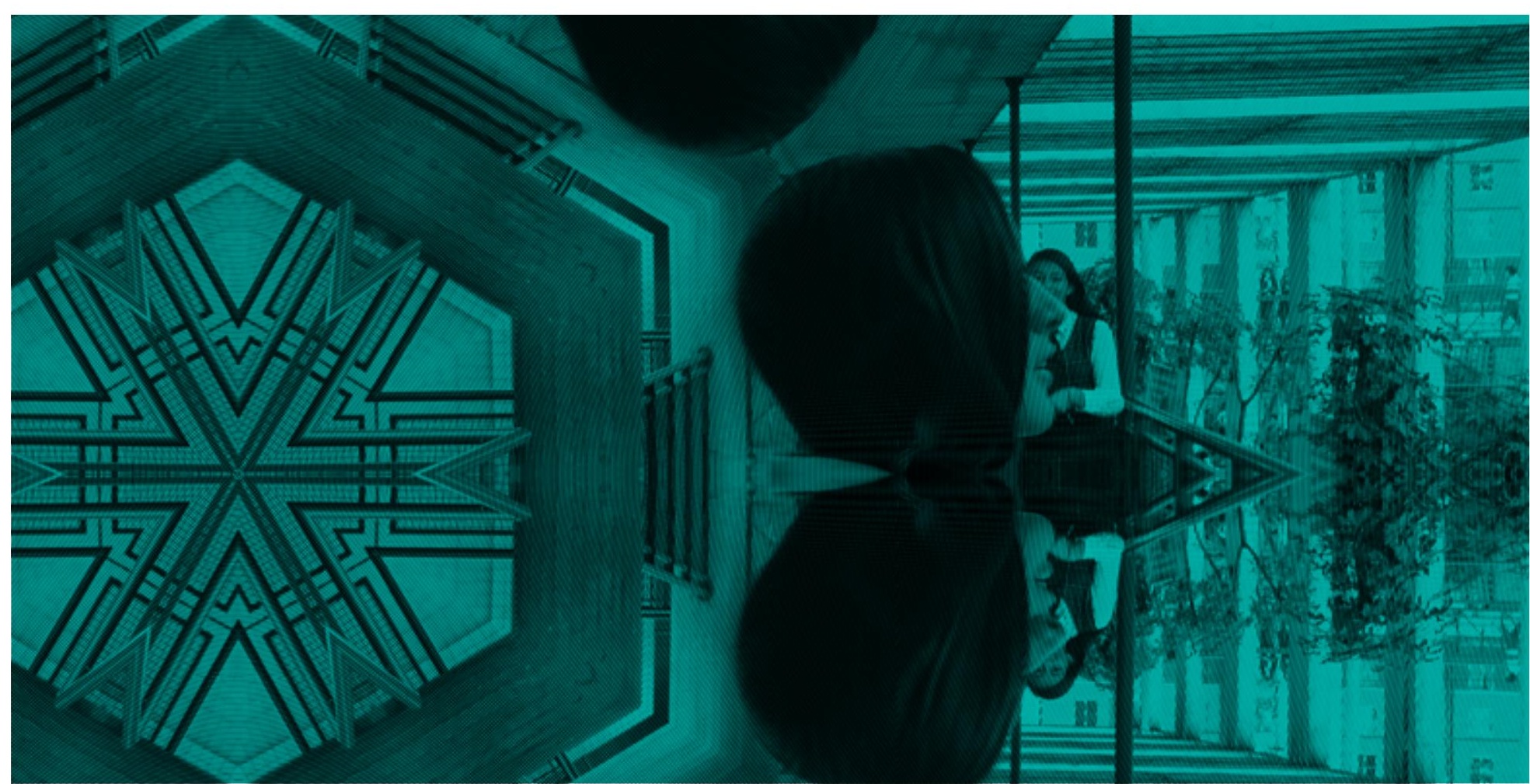


Para este artículo de reflexión se analizaron los datos de las entrevistas $^{8}$ a 21 docentes de la jornada mañana, y de las encuestas aplicadas a 99 estudiantes de cuatro cursos en el grado sexto, para establecer puntos comunes y diferencias en la manera como perciben las situaciones relacionadas con violencia escolar y el tratamiento de conflictos en su institución; el análisis se complementó con notas de campo a partir de la observación directa. Se elaboraron tablas y gráficos como apoyo al análisis y presentación de los hallazgos, tomando las muestras de manera proporcional ${ }^{9}$, y se acudió a respuestas literales de los participantes. La participación fue voluntaria y se mantiene absoluta reserva de los datos recogidos. Los resultados encontrados fueron socializados.

\section{Análisis de resultados}

En cuanto a la caracterización de los docentes, el 48\% de los entrevistados fueron hombres (diez), y el 52\% mujeres (once). Para 2013, laboraban cuarenta docentes y cuatro directivos docentes en la jornada de la mañana del colegio donde se llevó a cabo el diagnóstico; dieciocho docentes $(86 \%$ de la muestra) y tres directivos (el coordinador de convivencia, el rector y la orientadora) fueron entrevistados, preguntando por el año de ingreso a la entidad, pues se considera que este dato es relevante porque permite inferir qué tanto reconocen las problemáticas del lugar. Se observó la presencia de dos grupos mayoritarios de docentes: los que están desde el período de 1985-1995 y los que han llegado desde $2006^{10}$.

La encuesta fue aplicada a 99 estudiantes de grado sexto (60 niños y 39 niñas), cuyas edades oscilaban entre los 10 y 14 años. El $66 \%$ cursaba sexto por primera vez y el $30 \%$ lo hacía por segunda o tercera vez; un 3\% no informó sobre este ítem. Al preguntar a los participantes si habían sido testigos de manifestaciones de violencia escolar en algún escenario (salón de clases, lugares externos al colegio) y momentos de la jornada académica (descansos, cambios de clase), sus respuestas muestran porcentajes similares en los ítems de salón de clases y descansos (entre el 44\% y el 47\%); situación que cambia en los otros escenarios y momentos; pues los docentes perciben que hay mayores expresiones de violencia en los cambios de clase (62\%), lo cual podría explicarse

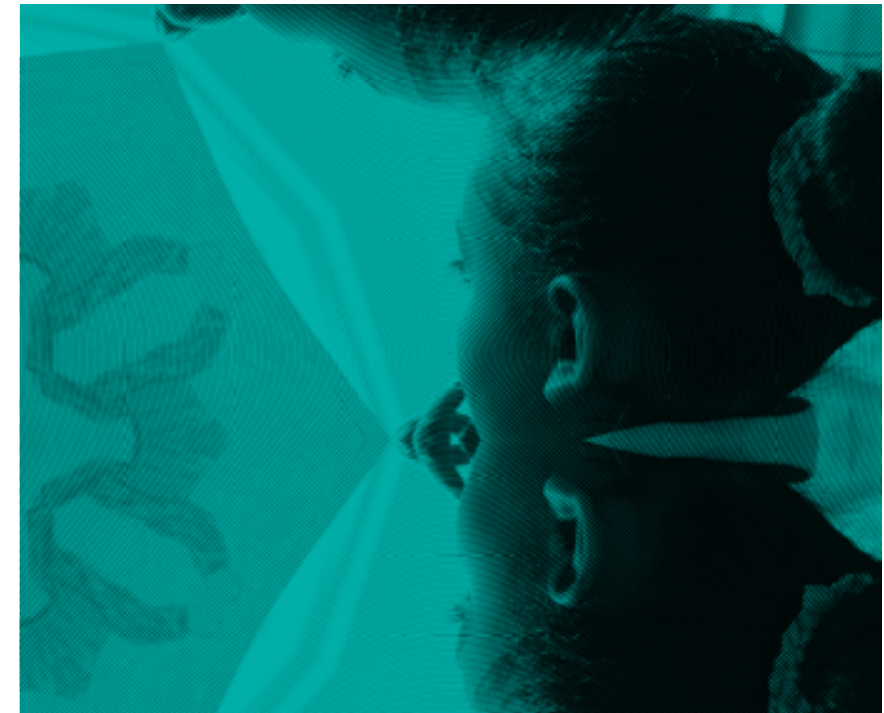

por el hecho de que ellos se movilizan más al culminar una clase, mientras que los estudiantes por lo general permanecen en sus salones (Gráfico 1).

Los participantes coinciden en declarar haber sido testigos de manifestaciones de violencia escolar por fuera de la institución, siendo más alta la percepción de los docentes (81\%), quienes además comentaron que hay estudiantes que se citan a las afueras del colegio para tramitar los conflictos a través de riñas. Estos resultados reflejaron la presencia de expresiones de violencia escolar en varios momentos y escenarios, lo que constituye un hecho preocupante por cuanto se idealiza la escuela como escenario pacífico y armónico, donde el interés primordial de los que allí asisten es llevar a cabo procesos de aprendizaje y convivencia solidaria. El siguiente gráfico presenta el contraste de las respuestas.

8 Las entrevistas a los docentes constaron de 5 preguntas abiertas y, con base en los hallazgos, se desarrolló una encuesta de siete preguntas (dos de ellas abiertas) que fue aplicada a los estudiantes.

9 En el caso de los profesores, se dividió el número de respuestas de cada ítem, entre 21; y las de los estudiantes entre 99.

10 El decreto de creación del Colegio es del año 1977. 


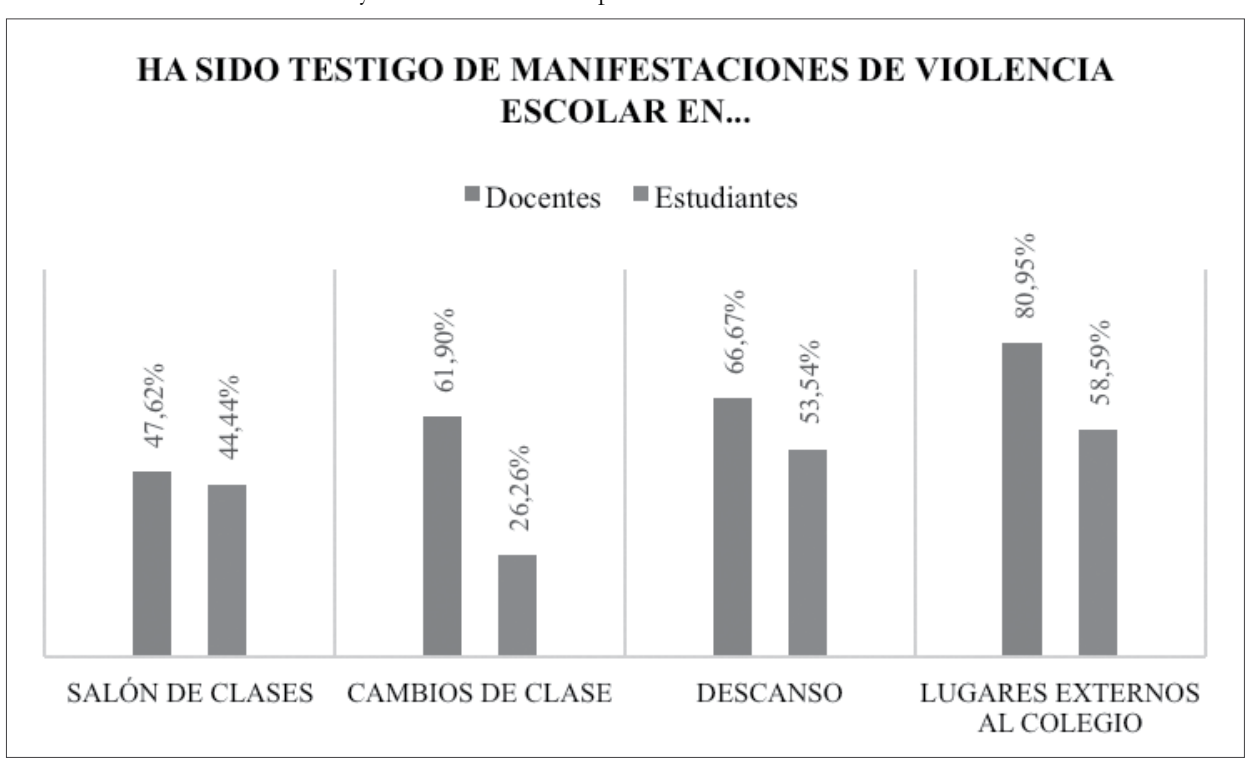

Fuente: Elaboración propia a partir de los hallazgos

Los participantes atribuyen a distintos problemas las manifestaciones de violencia escolar, siendo los porcentajes bastante dispares al realizar una comparación. Los docentes señalaron como principal problemática la falta de tolerancia $(57 \%)$, mientras que

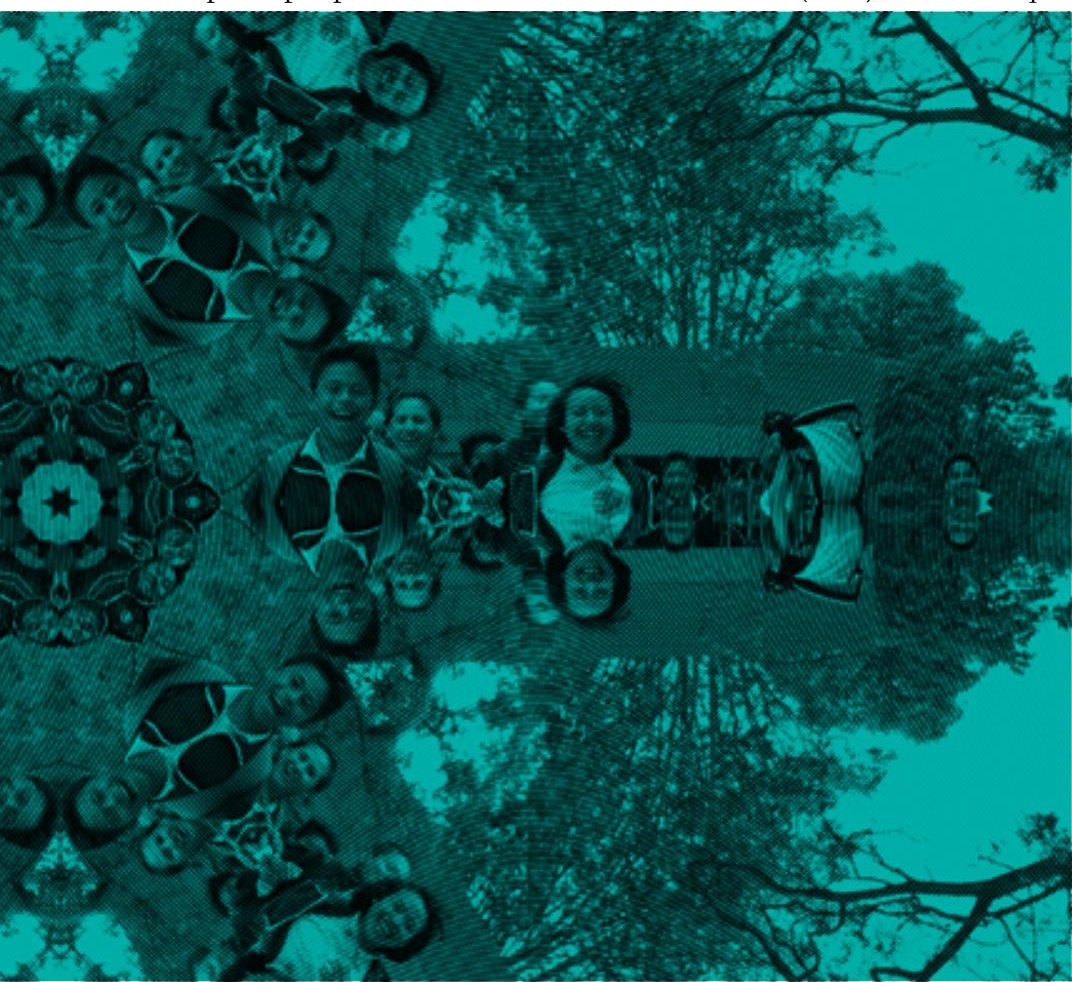

los estudiantes mencionaron el consumo de sustancias psicoactivas (SPA) y el matoneo (ambas con el 59,6\%), los malos entendidos $(49 \%)$ y el mal manejo de la ira. También aparecieron respuestas únicas en cada grupo: los docentes afirmaron que las peleas y la falta de control/autoridad institucional están relacionadas con esas expresiones; mientras que los estudiantes las vincularon con la presencia de barras bravas en la institución (Gráfico 2).

Las respuestas permiten ver que las miradas frente a la violencia escolar dependen del rol que se desempeñe en una institución educativa, por lo que al momento de generar estrategias contextualizadas para abordar eficazmente estas situaciones, se debe tener presente este hecho, valorando los puntos de vista de los actores implicados. Para los estudiantes, por ejemplo, el mal manejo de la ira fue la problemática más asociada a los brotes de violencia, alcanzando un $41 \%$, mientras que para los docentes fue de solo 9\%. Que los estudiantes den tal alta valoración expone la necesidad de trabajar aspectos relacionados con el autocontrol, estrategias de comunicación asertiva y de empatía, para reforzar positivamente la manera como los estudiantes reaccionan frente a un conflicto, claro está, desde un enfoque práctico y reflexivo. 


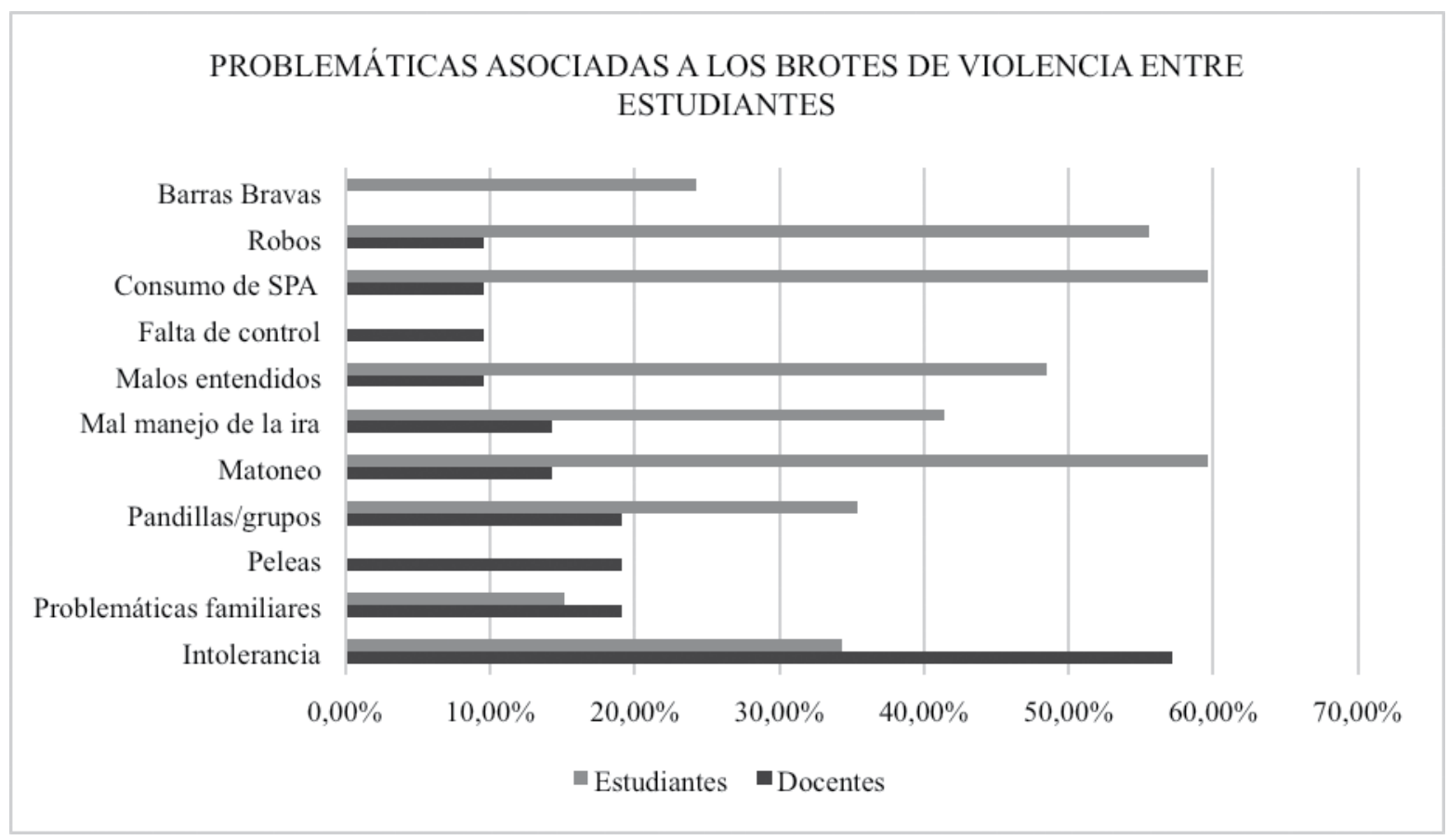

Fuente: Elaboración propia a partir de los hallazgos

Los docentes indican que los conflictos se resuelven con el uso de golpes y violencia física ( $81 \%$ ); mientras que para los estudiantes es más evidente la existencia de acuerdos (52\%) y la confrontación verbal (39\%). Es necesario considerar que los docentes trabajan con varios niveles, en este caso, los estudiantes de grado sexto no coinciden con su percepción de que los golpes o la violencia física son la manera más frecuente de tramitación. Los diálogos con mediación alcanzaron un porcentaje muy similar (19\%) para docentes y estudiantes, lo cual permite inferir que aunque no son la primera opción, son tenidos en cuenta por algunos estudiantes y esto se podría reforzar. Se percibe el uso de estrategias pacíficas de resolución, pero éstas requieren de la mediación de un tercero. Nuevamente las respuestas revelan diferencias importantes en las percepciones de los participantes (Gráfico 3). 


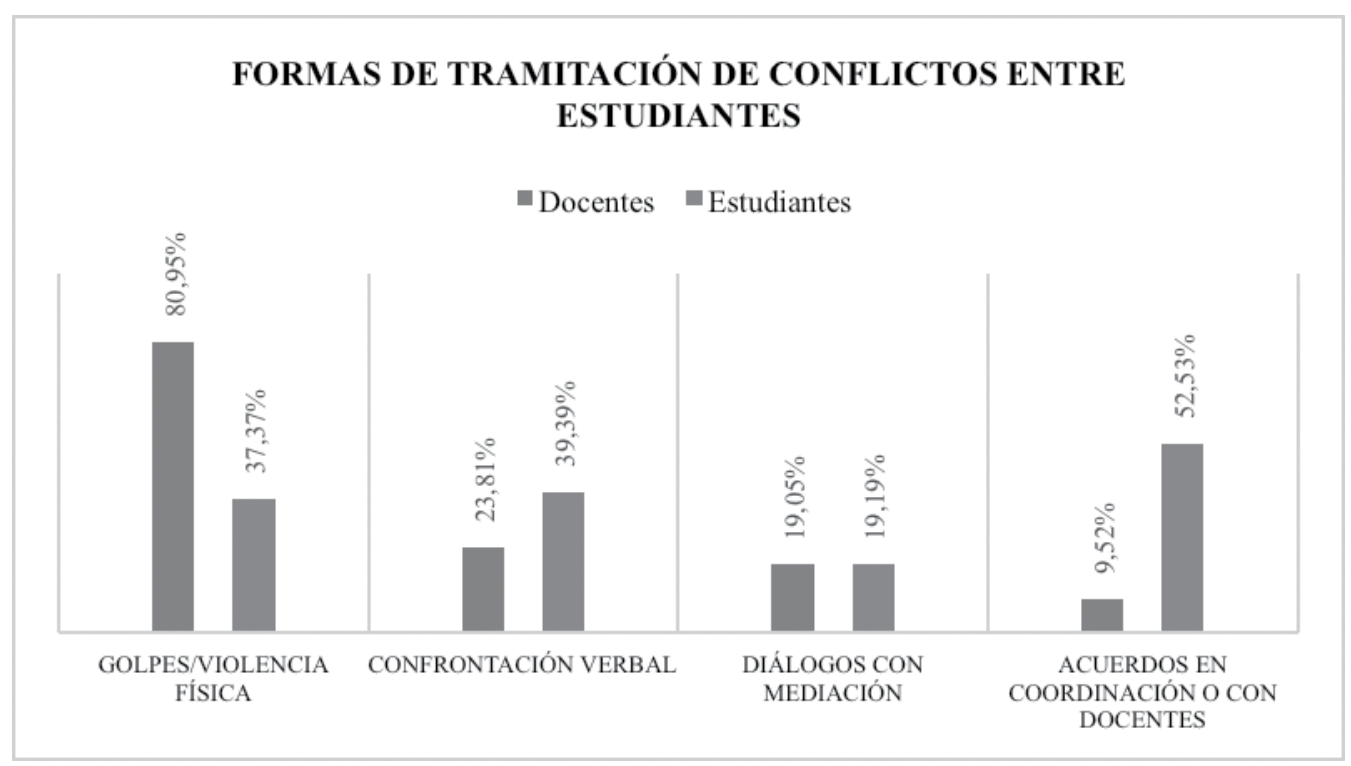

Fuente: Elaboración propia a partir de los hallazgos

$\mathrm{Al}$ preguntar por posibles estrategias para transformar esta situación al interior de la escuela, la mayoría de los docentes refirió estrategias como motivar la reflexión a través de talleres con padres y estudiantes (48\%); el trabajo con valores como el respeto, la tolerancia y la autoestima (24\%); la formación espiritual y la conformación de comités de mediadores en conflictos (29\%). Otras estrategias señaladas fueron la aplicación del manual de convivencia, la remisión a instancias internas ${ }^{11}$ o externas ${ }^{12}$ y la promoción de actos de justicia. Muy pocos se refirieron a sancionar o transformar las relaciones de poder evidentes en la estructura escolar.
El panorama es bastante diferente con los estudiantes, pues su opinión coincide muy poco con las estrategias pacíficas y pedagógicas señaladas por los docentes (entre el 3\% y 15\%); sus opciones se inclinaron especialmente por la expulsión o sanción (14\%), acciones que promuevan la vigilancia (30\%); y un constante acompañamiento de docentes $(20 \%)$, incluso hubo respuestas que sugerían "no hacer nada" (3\%). Un solo estudiante hizo énfasis en la posibilidad de un acto de reconciliación (Tabla 1).

11 Como por ejemplo Orientación escolar o Coordinación de Convivencia.

12 Instituto Colombiano de Bienestar Familiar, comisarías, centros de orientación juvenil e incluso la Policía. 
Tabla 1. Comparación de algunas estrategias de docentes y estudiantes frente a la violencia escolar

\begin{tabular}{|c|c|}
\hline Estrategias sugeridas por docentes & Estrategias sugeridas por estudiantes \\
\hline $\begin{array}{l}\text { - "Conformación de equipos conciliadores o mediadores } \\
\text { en cada ciclo educativo" (Directivo docente, género } \\
\text { femenino, labora desde 1989) } \\
\text { "Hacer tomar conciencia de la importancia de dialogar } \\
\text { y conciliar; aplicar la ley de convivencia" (Docente, } \\
\text { género femenino, labora desde 2007) } \\
\text { "Democratización de la escuela, cambiar las relaciones } \\
\text { de poder" (Docente, género masculino, labora desde } \\
2009, \text { ciclo cinco) } \\
\text { " "Educar primero a los padres" (Docente, género } \\
\text { femenino, labora desde 1991, ciclo tres) } \\
\text { - Sanciones que valgan la pena; más que una } \\
\text { estrategia pedagógica, se necesita que el estudiante } \\
\text { entienda que todo acto, positivo o negativo, tiene } \\
\text { consecuencias" (Docente, género femenino, labora } \\
\text { desde 2009, ciclo cuatro) }\end{array}$ & $\begin{array}{l}\text { - "Para disminuir la violencia escolar, que ayan } \\
\text { campeonato de bokseo para que toda la rabia contenida } \\
\text { y sacarla en el rin" (género femenino, } 14 \text { años, primera } \\
\text { vez en sexto) (sic). } \\
\text { - "No aseptar niños que en su observador se encuentren } \\
\text { aspectos muy malos, en otras palabras, que no asepten } \\
\text { a cualquier niño" (género masculino, } 11 \text { años, primera } \\
\text { vez en sexto) (sic) } \\
\text { "Que se hicieran campañas de la violencia y les } \\
\text { enseñaran a ser tolerantes, y más atención de los } \\
\text { profesores y la coordinadora" (género femenino, } 14 \\
\text { años, segunda vez en sexto) (sic) } \\
\text { "Más vigilamiento, más restricción, más celadores o } \\
\text { más cámaras para tener vigilado a los estudiantes" } \\
\text { (género masculino, } 12 \text { años, primera vez en sexto) } \\
\text { "Me gustaría más atención de los profesores hacia } \\
\text { los estudiantes para evitar más conflictos" (género } \\
\text { femenino, } 14 \text { años, tercera vez en sexto) (sic) }\end{array}$ \\
\hline
\end{tabular}

Fuente: Elaboración propia a partir de los hallazgos

Las estrategias planteadas por los docentes no contemplan intervenciones desde el interior de las áreas del plan de estudios, lo que permite concluir que entienden el problema de la violencia como un factor externo; sus estrategias estuvieron orientadas a acciones propias de los estudiantes, pero prevén muy poco la posible influencia del contexto social y de las relaciones interpersonales entre docentes y estudiantes. Solo un docente se refirió a "democratizar la escuela", hecho que tácitamente evidencia que las relaciones de poder pueden ser generadoras de violencia, tal como lo señalaba el IIDH (2011).

Llama la atención la solicitud reiterada de los estudiantes por una mayor vigilancia de sus docentes como medio efectivo para prevenir conflictos. Esto respalda las premisas de suponer el conflicto como hecho mediado por actos de otros; de no interiorizar y asumir el rol que pueden jugar, tanto en la aparición de esas manifestaciones violentas, como en su prevención y manejo. El siguiente gráfico expone una comparación de los hallazgos. 


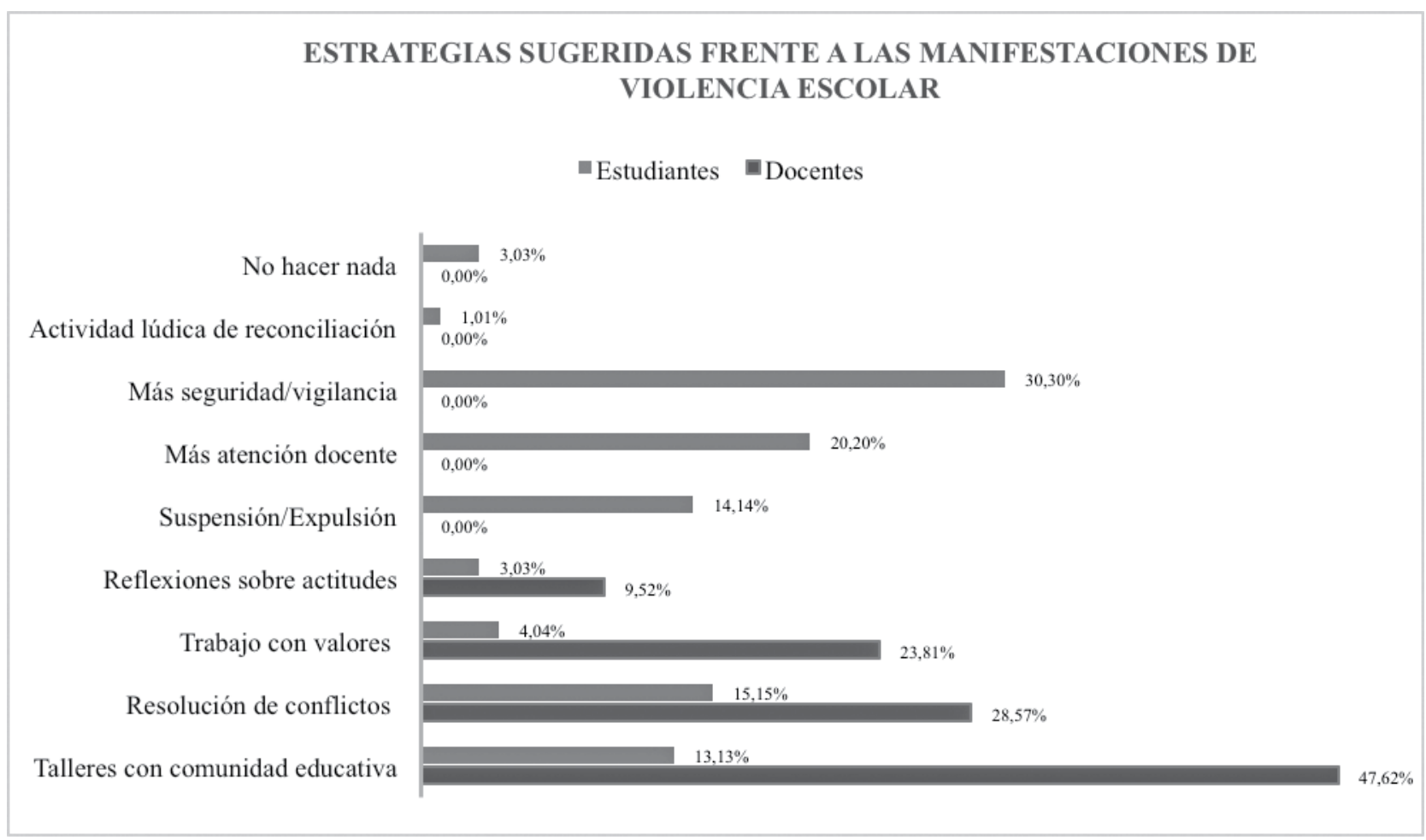

Fuente: Elaboración propia a partir de los hallazgos

\section{Hacia una convivencia pacífica en la escuela}

En las estrategias propuestas se destaca que los docentes asignan gran responsabilidad a las actitudes de los estudiantes y a la necesidad de apoyo de su familia. Al tiempo, fue usual la solicitud de los estudiantes de un otro que regule su conducta, que les vigile, acompañe y actúe tal vez como agente represor; lo cual permite cuestionar la manera en que se están acostumbrando a la intervención externa, antes de optar por una postura autónoma que desestime las expresiones de violencia.

Antes de trazar cualquier plan de intervención, es necesario comprender las expresiones que atentan contra una convivencia pacífica en la escuela, como las relacionadas con la violencia escolar, fenómeno complejo que implica agresiones físicas y simbólicas, y que puede tener consecuencias como el deterioro de las relaciones interpersonales, la pérdida del sentido de identidad con el proyecto de la escuela y la proliferación de antivalores que promueven el "todo vale", al no darle legitimidad al otro en el convivir; lo que genera, en últimas, un clima escolar no favorable para el aprendizaje.

Gracias al auge tecnológico de la sociedad actual, tenemos acceso a un volumen importante de información en distintos formatos; lo que hace indispensable reflexionar sobre una transformación de la escuela donde, tan importante como las actividades académicas, sea el enfocarse en la construcción de relaciones sociales desde la empatía, la solidaridad, el respeto y la tolerancia. Al respecto, Soliá y Graterol (2013) afirman que:

Hoy día el problema no es el conocimiento, ya que éste se encuentra a disposición de la ciudadanía mundial. La situación problemática que se debe resolver es el desarrollo de capacidades, valores y destrezas imprescindibles para convivir sanamente (p. 405). 
Por su parte, Maturana y Vignolo (2001) sostienen que la convivencia de los niños se da de acuerdo con los modelos y conductas que ven en los adultos. Esto pone de manifiesto que se requiere formar ciudadanos que abandonen conductas y prácticas que propician la violencia y vulneración de derechos; hecho que puede y debe ser fomentado en la familia, pero también en la escuela. Otro elemento fundamental es la percepción del conflicto y las formas de manejarlo. En este sentido, es preciso señalar que si no se logran acuerdos favorables a ambas partes cuando aparece un conflicto, se pueden dar actos de vulneración de derechos y de violación de la dignidad humana. Una percepción equivocada o insuficiente sobre el conflicto escolar promueve un manejo ineficaz del mismo, lo cual puede suscitar conductas contraproducentes para la convivencia pacífica.

En los resultados se evidenció que los estudiantes utilizan pocas herramientas que permitan manejar los conflictos de manera asertiva, lo que hace necesario educar bajo enfoques por el respeto a la diversidad y para favorecer escenarios de negociación y mediación. Además, es imprescindible que las estrategias para hacer frente a las problemáticas que atentan contra la convivencia pacífica en nuestras escuelas, no estén orientadas solamente hacia los estudiantes, sino que involucren también a docentes, padres y directivos; pues todos deben estar en capacidad de abordarlas de una manera asertiva.

En el caso particular de los docentes, es importante comprender que son a la vez objeto y sujeto de observación. Como sujetos, prestan atención y concretan acciones (no solo académicas) frente a sus estudiantes, y como objetos de observación, son examinados constantemente por los demás actores, por lo que su proceder puede influir en la manera como se reacciona frente a situaciones específicas de conflicto.

El deseo de imponer genera relaciones de poder; el deseo de compartir genera relaciones dialógicas [...] las relaciones dialógicas se basan en la comunicación que lleva a todos los sujetos implicados a compartir una acción, acuerdo, sentimiento o deseo (Soler y Flecha, 2010, p. 369).

La apuesta por una convivencia pacífica debe partir de este supuesto, sin dejar de lado que se deben tomar posturas críticas y reflexivas frente a las circunstancias que la afectan; tal como señala García (2012) al sugerir que aunque los docentes deben ser gestores de espacios de encuentro y diálogo frente a situaciones de conflicto, también deben conocer y evaluar aquellos elementos de la cultura escolar que potencian comportamientos agresivos en la escuela.
Ahora bien, el propósito de este trabajo no fue identificar quién está o no en lo correcto, sino contrastar las percepciones de docentes y estudiantes frente a una problemática como la violencia escolar, lo que adquiere relevancia por cuanto la convivencia pacífica debe ser manejada como un proyecto conjunto en construcción permanente, que para ello requiere la legitimación de las miradas de sus actores.

Además, es oportuno resaltar que la escuela no influye directamente en las situaciones de la vida cotidiana que rebasan su campo de acción, por lo que no se deben escatimar esfuerzos para propiciar, desde todos sus frentes, una trasformación positiva de la manera como se manejan los conflictos, motivando el reconocimiento y legitimización del otro como una vía necesaria para el encuentro, lo que posibilitaría, en términos de Maturana (2002), las relaciones sociales. Para Pérez (2001), las relaciones pacíficas no serán posibles

Si persisten la competencia desleal; el irrespeto; la intolerancia; la deslegitimación de lo diverso; la despreocupación por el bienestar ajeno; el deseo de borrar la alteridad. Acciones todas que deben replantearse desde la educación, ya que su tarea consiste en facilitar el desarrollo de las personas como seres humanos que se respetan a sí mismos y a los otros con consciencia social y ecológica, de modo que puedan actuar con responsabilidad y libertad en la comunidad a la que pertenecen (p. 83).

Ortega (2013) apunta a lo mismo cuando reflexiona que no puede haber educación sin relaciones de alteridad, por lo que se debe procurar que éstas no sean de dominio-imposición o de indiferencia, sino de respeto y reconocimiento del otro. Lograr una convivencia pacífica en la escuela, y en la sociedad, es posible si se trabaja por el exilio de prototipos que etiquetan y hieren; promoviendo una cultura donde se comprenda que los seres humanos merecen ser tratados con dignidad. No hay fórmulas, pero sí factores comunes: no es posible convivir pacíficamente si hay desprecio por el otro; si se irrespetan y desconocen sus maneras particulares de ver la vida, aunque no se compartan; si se opta por el egoísmo y el uso de violencia de cualquier tipo, lo cual incluye la permisividad e indiferencia frente a su utilización. 


\section{Referencias}

Allodi, M. (2010). Goals and values in school: a model developed for describing, evaluating and changing the social climate of learning environments. Soc Psychol Educ, pp. 207-235.

Arellano, N. (2007). La violencia escolar y la prevención de conflicto. Orbis/Ciencias Humanas. Año 3. No 7, pp. 23-45. Obtenido el 10 de Julio de 2014, desde http://www.redalyc.org/articulo.oa?id=70930703

Ávila, A., Bromberg, P., Pérez, B., y Villamil, M. (2014). Clima escolary victimización en Bogotá, 2013. Encuesta de convivencia escolar. Bogotá: Imprenta Nacional de Colombia. pp. 25, 30, 38, 215-219, 227-232.

Camargo, M. (1996). Violencia escolar y violencia social. Trabajo presentado en el Quinto Congreso Nacional de Prevención y Atención del Maltrato Infantil, noviembre, Bogotá. Obtenido en Octubre de 2013, desde http://www.acosomoral.org/pdf/rce34_03ensa.pdf

Castro, A. (2010). Desencontrándonos. Cuando no se educa para estar bien con los demás. Revista Internacional Magisterio, No. 44, pp. 10-15.

Congreso de la República de Colombia. (2013). Ley 1620 del 15 de maržo. Bogotá. Obteido en Noviembre de 2013, desde http://wsp.presidencia.gov.co/Normativa/Leyes/ Documents/2013/LEY\%201620\%20 DEL\%2015\%20DE\%20MARZO\%20DE\%202013.pdf

Dincyurek, S., y Civelek, A. (2008). The determination of the conflict resolution. Strategies of university students that they use when they have conflicts with people. Behavior Analyst Today, 9 (3-4), pp. 215-233. Obtenido en Septiembre de 2014, desde http:/ / files.eric.ed.gov / fulltext/EJ862245.pdf

García, B. (2012). Violencia y educación. Bogotá: Universidad Distrital Francisco José de Caldas.

García, B., Guerrero, J., y Ortiz, B. (2012). La violencia escolar en Bogotá desde la mirada de las familias. Doctorado Interinstitucional en Educación. No. 3. Serie Grupos. Bogotá: Universidad Distrital Francisco José de Caldas.

González, J., Villalobos, E., y Lauretti, P. (2009). Manejo y resolución de conflictos escolares. Revista Mexicana de Orientación Educativa - Remo,
VI(17), pp. 43-52. Obtenido en Octubre de 2014, desde http:/ /www. remo.ws/REVISTAS/remo-17.pdf

Henry, S. (2000). What is school violence? An Integrated Definition. Annals of the American Academy of Political and Social Science, No. 567, pp. 16-29. Obtenido en Marzo de 2015, desde http://www.jstor.org/ stable/1049491

IIDH. (2011). X Informe Interamericano de la Educación en Derechos Humanos. Desarrollo en las politicas de convivencia y seguridad escolar con enfoque de derechos. Instituto Interamericano de Derechos Humanos. San José, Costa Rica: Segura Hermanos S.A. Obtenido el 14 de Abril de 2015, desde http:/ / www.iidh.ed.cr/multic/UserFiles/Biblioteca/IIDH/9_2012/6860.pdf

Johnson, D., y Johnson, R. (1999). Cómo reducir la violencia en las escuelas. Buenos Aires: Paidós Educador, p. 29

Martínez, V., y Pérez, O. (2005). Conflictividad escolar y fomento de la convivencia. Revista Iberoamericana de Educación, No. 38. Obtenido en Octubre de 2014, desde http://rieoei.org/rie38a02.htm

Maturana, H. (2002). Emociones y lenguaje en educación y política. España: Dolmen.

Maturana, H., y Vignolo, C. (2001). Conversando sobre Educación. Perspectivas- Departamento de Ingeniería Industrial, Universidad de Chile, 4 (2), pp. 249-266. Obtenido en Noviembre de 2014, desde http://www.dii.uchile.cl/ revista/ArticulosVol4-N2/249-266\%2005-H.pdf

Mockus, A., y Corzo, J. (2003). Cumplir para convivir. Factores de convivencia y tipos de jóvenes por su relación con normas y acuerdos. Bogotá: Universidad Nacional de Colombia-Instituto de estudios políticos y relaciones internacionales. Unibiblos, p. 14.

Noguera, P. (1995). Preventing and producing violence: A critical analysis of responses to school violence. Harvard Educational Review, 65(2), pp. 189-212. Obtenido desde http://dx.doi.org/10.17763/ haer.65.2.e4615g5374044q28

Ortega, P. (2013). La pedagogía de la alteridad como paradigma de la educación intercultural. Revista Española de Pedagogía, LXXI (256), pp. 
401-422. Obtenido en Noviembre de 2014, desde https:/ / dialnet.unirioja.es/servlet/articulo?codigo $=4391820$

Pérez, T. (2001). Convivencia solidaria y democrática. Nuevos paradigmas y estrategias pedagógicas para su construcción. Bogotá: Fotolito América, p. 83.

Powell, M., y Ladd, L. (2010). Bullying: A review of the literature and implications for family therapists. The American Journal of Family Therapy (38), pp. 189-206.
Soler, M., y Flecha, R. (2010). Desde los actos de habla de Austin, a los actos comunicativos. Perspectivas desde Searle, Habermas y CREA. Revista Signos, 43(2), pp. 363-375. Obtenido en Septiembre de 2015, desde http://dx.doi.org/10.4067/S0718-09342010000400007

Soliá, R., y Graterol, N. (2013). La alteridad como puente para la trascendencia ética. TELOS. Revista de Estudios Interdisciplinarios en Ciencias Sociales Universidad Rafael Belloso Chacin, 15(3), pp. 400-413. Obtenido en Noviembre de 2014, desde http://www.redalyc.org/pdf/993/99328424008.pdf 